\title{
A mobilidade ativa e os Objetivos de Desenvolvimento Sustentável 2030, com foco na educação para a saúde e para o meio ambiente: Uma análise a partir das características do indivíduo, do ambiente e da tarefa
}

\author{
Active mobility and the 2030 Sustainable Development Goals, with a focus on education for health
} and the environment: An analysis from the characteristics of the individual, the environment and

the task

Movilidad activa y Objetivos de Desarrollo Sostenible 2030, enfoque en educación para el la salud, y educación ambiental: Un análisis desde las características del ambiente, el individuo y la tarea

Recebido: 13/05/2021 | Revisado: 19/05/2021 | Aceito: 21/05/2021 | Publicado: 07/06/2021

Itamar Adriano Tagliari
ORCID: https://orcid.org/0000-0002-1479-8282
Universidade Estadual de Ponta Grossa, Brasil
Secretaria de Estado do Desenvolvimento Sustentável e do Turismo, Brasil
E-mail: itagliari@ig.com.br
Urivald Pawlowsky
ORCID: https://orcid.org/0000-0003-1113-1201
Universidade Federal do Paraná, Brasil
E-mail:urpawl@gmail.com

\begin{abstract}
Resumo
O objetivo deste artigo é verificar a mobilidade ativa e os Objetivos de Desenvolvimento Sustentável 2030, com foco na educação para a saúde e para o meio ambiente, a partir das características do indivíduo, do ambiente e da tarefa. O estudo foi do tipo descritivo documental. Foi analisado o documento intitulado "Transformando Nosso Mundo: A Agenda 2030 para o Desenvolvimento Sustentável” (Organização das Nações Unidas [ONU], 2015). Categorias de análise: saúde, educação e mudanças climáticas. As categorias foram analisadas a partir das características do indivíduo, do ambiente e da tarefa, e suas interconexões, propostas pelo Modelo de Newel (Newell \& Jordan, 2007; Newell, 1986). Os resultados indicam que a mobilidade ativa apresenta-se como um meio para a promoção da saúde e para diminuir a quantidade de gases poluentes do meio ambiente. $\mathrm{O}$ ambiente de aprendizagem educacional cria oportunidades, instrução e encorajamento para que a mobilidade ativa seja efetuada de forma saudável e em um ambiente sustentável. Concluiu-se que a mobilidade ativa pode ser desenvolvida no ambiente educacional formal e não formal, em atividades pontuais, bem como por meio de intervenções de manutenção a longo prazo, além disso a ampliação dos contextos de ações são necessários, visando maior efetividade na saúde e no meio ambiente.
\end{abstract}

Palavas-chave: Desenvolvimento motor; Regras; Bicicleta.

\begin{abstract}
The purpose of this article is to verify active mobility and the 2030 Sustainable Development Goals, focusing on education for health and the environment, based on the characteristics of the individual, the environment and the task. The study was of the descriptive documentary type. The document entitled "Transforming Our World: The 2030 Agenda for Sustainable Development" (United Nations [UN], 2015) was analyzed. Analysis categories: health, education and climate change. The categories were analyzed based on the characteristics of the individual, the environment and the task, and their interconnections, proposed by the Newel Model (Newell \& Jordan, 2007; Newell, 1986). The results indicate that active mobility presents itself as a way of promoting health and reducing the amount of polluting gases in the environment. The educational learning environment creates opportunities, instruction and encouragement for active mobility to be carried out in a healthy way and in a sustainable environment. It was concluded that active mobility can be developed in the formal and non-formal educational environment, in specific activities, as well as through long-term maintenance interventions, in addition to expanding the contexts of the actions that are necessary, aiming at greater effectiveness in health and the environment.
\end{abstract}

Keywords: Motor development; Rules; Bicycle.

\section{Resumen}

El propósito de este artículo es verificar la movilidad activa y los Objetivos de Desarrollo Sostenible 2030, centrándose 
en la educación para la salud y el medio ambiente, a partir de las características del individuo, el ambiente y la tarea. El estudio fue de tipo documental descriptivo. Se analizó el documento titulado "Transformar nuestro mundo: La Agenda 2030 para el Desarrollo Sostenible" (Naciones Unidas [ONU], 2015). Categorías de análisis: salud, educación y cambio climático. Las categorías se analizaron en función de las características del individuo, el entorno y la tarea, y sus interconexiones, propuestas por el Modelo Newel (Newell \& Jordan, 2007; Newell, 1986). Los resultados indican que la movilidad activa se presenta como un medio para promover la salud y reducir la cantidad de gases contaminantes en el medio ambiente. El entorno de aprendizaje educativo genera oportunidades, instrucción y incentivos para que la movilidad activa se lleve a cabo de forma saludable y en un entorno sostenible. Se concluyó que la movilidad activa se puede desarrollar en el ámbito educativo formal y no formal, en actividades específicas, así como a través de intervenciones de mantenimiento a largo plazo, además de ampliar los contextos de acciones que sean necesarias, apuntando a una mayor efectividad en salud y medio ambiente.

Palabras clave: Desarrollo motor; Reglas; Bicicleta.

\section{Introdução}

O transporte ativo envolve os meios de locomoção à propulsão humana, tais como: pedestres, bicicletas, triciclos, patins, skates e cadeiras de rodas (Carvalho \& Freitas, 2012). "Além de não gerar poluição atmosférica e sonora, ele ocupa menos espaço físico que os automóveis, menor custo econômico, aproxima as pessoas ao invés de segregar e oferece a oportunidade de praticar atividade física sem que o indivíduo tenha que dispor um tempo extra do seu dia para isto" (Carvalho \& Freitas, 2012, p. 1618).

O transporte ativo, neste artigo, tratado como a mobilidade ativa, vem incrementar o transporte como meio de desenvolvimento sustentável, visto que utiliza o próprio corpo humano como propulsão, realizando a atividade física, contribuindo assim para a saúde humana, e não emitindo gases poluentes, favorecendo o meio ambiente. Segundo Edra (2019, p.10), "[o]s custos de saúde e ambientais são grandes, daí ser crucial repensar as cidades de forma mais inteligente, permitindo o maior uso da bicicleta para locomoção diária."

O desenvolvimento sustentável vem sendo abordado pela Organização das Nações Unidas por meio da Agenda 2030, que reúne os 17 Objetivos de Desenvolvimento Sustentável (ODS) e suas 169 metas (ONU, 2015). Eles correspondem ao plano de ação que orientará até o ano 2030 os trabalhos das Nações Unidas e dos países rumo ao desenvolvimento sustentável. Entre os ODS são tratadas questões relacionadas a saúde, ao meio ambiente e a educação, tais como nos seguintes objetivos de desenvolvimento sustentável: ODS 3, envolve a vida saudável e o bem-estar para todos, em todas as idades e o ODS 13, visa combater a mudança climática e seus impactos. O ODS 4, trata da educação inclusiva e equitativa e de qualidade, e promover oportunidades de aprendizagem ao longo da vida para todos (ONU, 2015).

Observam-se nesses ODS a preocupação em estimular o desenvolvimento humano e um meio ambiente que favoreça esse desenvolvimento, o que é uma das características do Modelo de Newell (Newell, 1986). Esse autor apresenta seu modelo composto de três fatores: i) restrições do indivíduo; ii) restrições do ambiente; iii) restrições da tarefa; e as suas interconexões. Ressalta-se que a restrição é uma característica do indivíduo, do ambiente ou da tarefa que encoraja alguns movimentos, enquanto desencoraja outros.

Conforme o Modelo de Newell, as restrições do indivíduo são estruturais ou funcionais, trata-se de características físicas e mentais que distinguem as pessoas, tornando-as únicas. As restrições estruturais estão relacionadas à estrutura corporal e as restrições funcionais estão relacionadas ao comportamento. As restrições da tarefa referem-se à estrutura, ao objetivo e à complexidade da tarefa, suas regras e estratégias. Por sua vez, as restrições do ambiente referem-se ao mundo que nos cerca, tais como as superficies do ambiente e mudanças climáticas, bem como as condições socio culturais que abrangem a oportunidade, a instrução e o encorajamento para a prática (Newell \& Jordan, 2007). Ainda em se tratando das características do ambiente temos o contexto que, para Bronfenbrenner (1996), envolve o estudo científico da acomodação progressiva, mútua, entre um ser humano ativo, em desenvolvimento, e as propriedades mutantes dos ambientes imediatos em que a pessoa em desenvolvimento vive, conforme esse processo seja afetado pelas relações entre esses ambientes, e pelos contextos mais amplos em que os 
ambientes estão inseridos.

A educação apresenta-se como poderoso meio para o desenvolvimento humano saudável e do meio ambiente sustentável. É por meio da oportunidade, da instrução e do encorajamento que poderemos investir no desenvolvimento das caracteristicas do indivíduo, oferecendo tarefas adequadas às características do indivíduo e do ambiente.

Considerando o exposto, o problema abordado por este artigo é: como a mobilidade ativa se relaciona com os Objetivos de Desenvolvimento Sustentável 2030, com foco na educação para a saúde e para o meio ambiente, a partir das características do indivíduo, do ambiente e da tarefa? O objetivo é verificar a mobilidade ativa e os Objetivos de Desenvolvimento Sustentável 2030, com foco na educação para a saúde e para o meio ambiente, a partir das características do indivíduo, do ambiente e da tarefa.

\section{Metodologia}

A presente pesquisa classifica-se como descritiva documental. Tem cunho descritivo, pois procurou descobrir, analisar e comparar associações entre variáveis e também por fazer uso de técnicas padronizadas para a coleta de dados. Com base nos procedimentos técnicos utilizados, este estudo tem caráter documental, considerando que buscou seus dados em documentos que não tiveram um tratamento analítico, chamados de documentos de "primeira ou segunda mão" (Gil, 2002).

Neste estudo analisou-se o documento intitulado "Transformando Nosso Mundo: A Agenda 2030 para o Desenvolvimento Sustentável" (ONU, 2015). As categorias de análise foram os seguintes Objetivos de Desenvolvimento Sustentável (ODS) (ONU, 2015): Objetivo 3 (ODS 3) - Vida saudável e o bem-estar para todos, em todas as idades; Objetivo 4 (ODS 4) - Educação inclusiva e equitativa e de qualidade, e promover oportunidades de aprendizagem ao longo da vida para todos; Objetivo 13 (ODS 13). Combater a mudança climática e seus impactos.

As categorias foram analisadas a partir das características do indivíduo (aptidão física e comportamento motor), do ambiente (oportunidade e estimulação educacional; mudanças climáticas; contexto) e da tarefa (complexidade, equipamento, objetivo e a estrutura de regras), e suas interconexões. As características do indivíduo, do ambiente e da tarefa, bem como suas interconexões, são propostas pelo Modelo de Newell (Newell \& Jordan, 2007; Newell, 1986).

\section{Resultados}

No Quadro 1 verificam-se os Objetivos de Desenvolvimento Sustentável 2030 (ODS) (ONU, 2015). São apresentados aqueles que fazem parte das categorias de análise deste estudo, a saber: ODS 3; ODS 4; e ODS 13.

Quadro 1 - Objetivos de Desenvolvimento Sustentável 2030: ODS 3; ODS 4; e ODS 13.

\begin{tabular}{|l|l|}
\hline $\begin{array}{l}\text { Objetivos de Desenvolvimento Sustentável } \\
\text { (ODS) 2030 }\end{array}$ & Descrição dos ODS 2030 \\
\hline Objetivo 3 (ODS 3) & Vida saudável e o bem-estar para todos, em todas as idades. \\
\hline Objetivo 4 (ODS 4) & $\begin{array}{l}\text { Educação inclusiva e equitativa e de qualidade, e promover } \\
\text { oportunidades de aprendizagem ao longo da vida para todos. }\end{array}$ \\
\hline Objetivo 13 (ODS 13) & Combater a mudança climática e seus impactos. \\
\hline
\end{tabular}

Fonte: Adaptado da ONU (2015).

No Quadro 1 tem-se a variável Objetivo de Desenvolvimento Sustentável 3 (ODS 3). Essa variável é subdividida em metas, entre elas destacamos, para fins deste estudo a meta 3.9, que versa sobre reduzir substancialmente o número de mortes e de doenças por poluição do ar (ONU, 2015). 
A segunda variável é o Objetivo de Desenvolvimento Sustentável 4 (ODS 4). Essa variável é subdividida em metas, entre elas destacamos, para fins deste estudo a meta 4.7, que visa garantir que todos os alunos adquiram conhecimentos e habilidades necessárias para promover o desenvolvimento sustentável, inclusive, entre outros, por meio da educação para o desenvolvimento sustentável e estilos de vida sustentáveis (ONU, 2015).

A terceira variável consiste no Objetivo de Desenvolvimento Sustentável 13 (ODS 13). Essa variável é subdividida em metas, entre elas destacamos, para fins deste estudo: a meta 13.3, que visa melhorar a educação, aumentar a conscientização e a capacidade humana e institucional sobre mitigação, adaptação, redução de impacto e alerta precoce da mudança do clima (ONU, 2015).

\section{Discussão}

A discussão dos resultados, inicialmente, será efetuada a partir da mobilidade ativa e dos Objetivos de Desenvolvimento Sustentável 2030 (ONU, 2015) com a saúde (ODS 3), depois com o meio ambiente (ODS 13), por fim com foco na educação (ODS 4) para a saúde e para o meio ambiente. Para a discussão dos resultados as variáveis serão analisadas a partir das categorias designadas como características do indivíduo, do ambiente e da tarefa, e suas interconexões (Newell \& Jordan, 2007; Newell, 1986).

No Quadro 1 apresenta-se a variável ODS 3, referente à vida saudável e ao bem-estar para todos, em todas as idades. Este assunto é tratado no Relatório Nacional Voluntário sobre os Objetivos de Desenvolvimento Sustentável (Brasil, 2017), indicando que a atividade física figura como componente importante no combate a doenças crônico-degenerativas da população, como a hipertensão e o diabetes.

Em se tratando das características do indivíduo, temos os fatores da aptidão física relacionados à saúde, que podem ser estimulados por meio da mobilidade ativa. Entre os fatores da aptidão física relacionados à saúde estão a composição corporal, a força, a potência e a resistência muscular, a resistência cardiorrespiratória, entre outros (Bouchard, Shepard, \& Stephens,1993).

Outra característica do indivíduo é o comportamento motor relacionado à mobilidade ativa. A interação do indivíduo, da tarefa e do ambiente modifica o comportamento motor, levando ao desenvolvimento motor (Newell, 1986). Gallahue e Donnely (2008) apontam para a importância da variabilidade de movimento durante o desenvolvimento motor, destacando: a) a quantidade: i) força: forte, leve, moderado; ii) tempo: rápido, lento, médio, estável, súbito; b) o espaço: i) níveis: alto, médio, baixo; ii) direções: para frente/para trás, diagonal, para cima/para baixo, curva, reta, zigue-zague; e c) os relacionamentos: i) objetos: ii) pessoas.

A realização da atividade física por meio da mobilidade ativa, seja como pedestre ou utilizando bicicletas, triciclos, patins, skates, cadeiras de rodas, entre outros, favorecerá a aptidão física e o desenvolvimento motor do indivíduo, que por sua vez oportunizará a prática da mobilidade ativa de formas cada vez mais satisfatórias.

Por outro lado, o sedentarismo é um dos problemas relacionados à obesidade, doença que acomete cada vez mais pessoas. A obesidade se caracteriza pelo excesso de tecido adiposo e se torna fator de risco para doenças como câncer, diabetes e problemas cardiovasculares (World Health Organization, 2013). Para a Organização Mundial da Saúde (World Health Organization, 2018), a atividade física regular de intensidade moderada - como andar de bicicleta - traz benefícios significativos para a saúde.

A aptidão física e as condições motoras do indivíduo são fundamentais para a realização da atividade física de forma segura e saudável, por meio da tarefa mobilidade ativa. Destaca-se, por exemplo, que a mobilidade ativa caminhar difere da mobilidade andar de bicicleta, visto que esta última envolve um equipamento que requer um aprendizado específico para ser utilizado. Além disso, as tarefas podem se tornar mais complexas se considerarmos o ambiente onde elas serão realizadas. 
Assim, para a realização da tarefa mobilidade ativa, no trânsito, além do conhecimento e a capacidade prática para a mobilidade ativa, a compreensão do Código de Trânsito (Brasil, 2020) torna-se imprescindível, visto que se constitui elemento de regras a serem seguidas na tarefa mobilidade ativa, visando o cuidado com a segurança e com a saúde humana. Nele estão as informações relativas aos equipamentos, objetivos e estrutura de regras para a realização da mobilidade ativa no trânsito. Contudo, para fins deste artigo, não trataremos das especificidades da prática da atividade física no trânsito.

A utilização da bicicleta, patins, triciclo e skate requerem estratégias específicas, o que as tornam complexas para o aprendiz, uma vez que tem que cuidar com a condução do meio de transporte e ao mesmo tempo respeitar a legislação do contexto que a circunda, o que requer uma educação para tanto. Em se tratando das características do ambiente, criar um contexto favorável para a oportunidade, instrução e o encorajamento da mobilidade ativa são fundamentais. Ainda em se tratando do ambiente, bem como da tarefa, no contexto das políticas públicas, o Conselho Federal de Educação Física (2012), por meio da Resolução CONFEF 229/2012, evidencia a necessidade de oferecer oportunidades por meio de programas, ações e estratégias de promoção, manutenção e recuperação da saúde e de prevenção de doenças.

Waters et al. (2011), em revisão sistemática, apontam a necessidade do desenvolvimento de intervenções que possam ser incorporadas à prática e aos sistemas em andamento, em vez de implementar intervenções que consumam muitos recursos e não possam ser mantidas por longo tempo. Além disso, conhecer quais componentes de intervenção específicos são mais eficazes e o que é acessível e custo-efetivo, é de grande importância para decisões a serem tomadas, e as avaliações econômicas devem aparecer em futuras pesquisas de prevenção da obesidade, se quisermos permitir decisões bem informadas sobre quais intervenções justificam a implementação em toda a população.

O padrão de utilização da bicicleta como meio de transporte ocorre de forma bastante heterogênea, mas potencialmente com maiores impactos nos países em desenvolvimento, onde se torna urgente a inclusão deste tema nas agendas de pesquisas sobre a relação entre a promoção do transporte ativo, a saúde e a segurança no trânsito (Carvalho \& Freitas, 2012).

A variável ODS 3, mais especificamente na meta 3.9, versa sobre reduzir substancialmente o número de mortes e doenças por poluição do ar. Assim, incentivar a tarefa mobilidade ativa visa também a diminuir a quantidade de meios de transporte que emitem gases poluentes do meio ambiente.

O desenvolvimento sustentável favorece a saúde humana, enfim, favorece as características ambientais que, por sua vez, favorecem as características do indivíduo. Segundo o Ministério da Saúde (Brasil, s/d), a exposição humana a poluentes atmosféricos, em pessoas que vivem em regiões metropolitanas, bem como em locais mais expostos aos poluentes, pode provocar impactos à saúde de acordo com a forma de exposição (aguda ou crônica), podendo agravar doenças preexistentes e/ou aumentar o número de casos de doenças respiratórias, oculares e cardiovasculares.

No Quadro 1, sobre o meio ambiente, tem-se a variável ODS 13, que visa tomar medidas urgentes para combater a mudança climática e seus impactos. Para tanto, ela apresenta diferentes metas, e para fins deste artigo definimos os seguintes: a meta 13.3, que propõe sobre melhorar a educação, aumentar a conscientização e a capacidade humana e institucional sobre mitigação, adaptação, redução de impacto e alerta precoce da mudança do clima. "A natureza global da mudança do clima requer a maior cooperação internacional possível visando a acelerar a redução das emissões globais de gases de efeito de estufa e abordar a adaptação aos impactos negativos das mudanças climáticas.” (ONU, 2015, p. 8).

Além disso, em estudo tratando das características do ambiente, Heaney, Carrión, Burkart, Lesk, e Jack (2019) verificaram os efeitos indiretos das mudanças climáticas sobre a saúde causados por alterações nos comportamentos de atividade física. O estudo forneceu evidências de que temperaturas acima de $28{ }^{\circ} \mathrm{C}$ estão associadas a declínios no comportamento de ciclismo da população na cidade de Nova Iorque. Os resultados são provavelmente específicos para esta área e tipo de programa de compartilhamento de bicicletas; trabalhos futuros devem investigar como essas relações variam em diferentes climas e 
estruturas sociais. Dado o papel crítico da atividade física na saúde humana, é crucial entender como as mudanças climáticas irão modificar a atividade física.

Observando a interação entre as características do indivíduo, do ambiente e da tarefa, verifica-se a necessidade de desenvolver as características físicas e motoras do indivíduo, visando a sua saúde e preparando-o para a tarefa mobilidade ativa. A mobilidade ativa, por sua vez, favorecerá o desenvolvimento de um meio ambiente saudável e sustentável, que contribuirá para a saúde humana.

Nesse sentido, a educação torna-se primordial. No Quadro 1, tem-se a variável ODS 4, que trata de assegurar a educação inclusiva e equitativa e de qualidade, e promover oportunidades de aprendizagem ao longo da vida para todos. Uma das metas desta variável é 4.7, que visa garantir que todos os alunos adquiram conhecimentos e habilidades necessárias para promover o desenvolvimento sustentável, inclusive, entre outros, por meio da educação para o desenvolvimento sustentável e estilos de vida sustentáveis.

Os ambientes de aprendizagem educacional formal e não formal, criando oportunidades e instrução e encorajamento, podem incluir as características do indivíduo, tais como as capacidades físicas, motoras e cognitivas da pessoa para a utilização dos meios de transporte ativo, que é uma tarefa que contém a complexidade, os equipamentos, os objetivos e a estrutura de regras próprias, para atuar de forma adequada em diferentes contextos ambientais. Assim, aprender a andar de bicicleta, skate, entre outros meios não motorizados, torna-se necessário, bem como nos meios motorizados. É importante fortalecer cada vez mais no ambiente escolar inicial, médio e universitário o aprendizado da mobilidade ativa.

Em se tratando da educação para a saúde, temos atividades oferecidas no ambiente formal educacional, nas aulas de Educação Física; e informal, em ambientes públicos e privados. Essas aulas instruem o aluno para a prática da atividade física, bem como sobre a sua importância para a saúde humana. Ao tratarmos da ODS 3 verificamos os benefícios da mobilidade ativa para a saúde humana. No ambiente escolar, é importante inserir nessas aulas assuntos relativos à mobilidade ativa em diferentes contextos, tais como no trânsito municipal, nas rodovias, nas estradas rurais, bem como em contextos com suas regras específicas, tais como clubes campestres, parques municipais, estaduais e nacionais, assuntos tanto teóricos como práticos. Contudo não trataremos das especificidades destes contextos neste artigo. A bicicleta é apresentada nos Parâmetros Curriculares Nacionais: Educação Física (Brasil, 1997), contudo, pode ser acrescentada nas escolas de maneira mais ampla, nas aulas de Educação Física. Mais importante é integrar os conteúdos da Educação Física com os diferentes contextos de prática. Embora o custo da compra e manutenção das bicicletas possa ser, a princípio, desencorajador, no entanto, seu retorno a longo prazo, se considerarmos seus benefícios, seria compensatório, visto diminuir o custo com a saúde e o meio ambiente. Além do contexto educacional formal, torna-se importante integrá-lo com o ambiente não formal, tanto público como o privado, para a prática da bicicleta e de outros meios de mobilidade ativa, como skate, entre outros, são importantes.

Já sobre a educação ambiental, a lei 9.795, de 27 de abril de 1999 (Brasil, 1999), destacam-se os meios pelos quais o indivíduo e a coletividade constroem valores sociais, conhecimentos, habilidades, atitudes e competências voltadas para a conservação do meio ambiente.

Existem programas e projetos em nível nacional, estadual e municipal. No âmbito nacional temos o Programa Nacional de Educação Ambiental (ProNEA) (Brasil, 2018). Temos também os projetos em esfera estadual, tais como verifica-se nos estados a seguir: O Programa Estadual de Educação Ambiental do Paraná (PEEA-PR), Secretaria de Estado do Desenvolvimento Sustentável e do Turismo (SEDEST) (Governo do Estado do Paraná, 2021), tem como principal objetivo ser referência para o planejamento e implementação de ações relativas à Política Estadual de Educação Ambiental, estabelecida pela Lei Estadual $n^{\circ}$ 17.505/2013 e regulamentada pelo Decreto no 9.958/2014. O Portal de Educação Ambiental reúne em seu endereço eletrônico informações sobre políticas públicas do setor de meio ambiente. No Portal do Estado de São Paulo é possível acessar o conteúdo 
sobre o meio ambiente, as práticas para uma vida sustentável, publicações, localizar acervos temáticos, assim como a programação das atividades oferecidas pelos órgãos do Sistema (Governo do Estado de São Paulo, 2020).

Entender a educação para saúde e para o meio ambiente, por meio da mobilidade ativa, de forma sistêmica, torna-se necessário. Bronfenbrenner (1996) explica a importância de entendermos o contexto de forma sistêmica. Seu modelo envolve o estudo científico da acomodação progressiva, mútua, entre um ser humano ativo, em desenvolvimento, e as propriedades mutantes dos ambientes imediatos em que a pessoa em desenvolvimento vive, conforme esse processo é afetado pelas relações entre esses ambientes e pelos contextos mais amplos em que os ambientes estão inseridos.

O mesmo autor informa que o ambiente mais imediato é o microssistema, ele é um padrão de atividades, papéis e relações interpessoais experienciados pela pessoa em desenvolvimento num dado ambiente com características físicas e materiais específicas. Um mesossistema inclui as interconexões entre dois ou mais microssistemas nos quais a pessoa em desenvolvimento participa ativamente. Destacam-se quatro tipos de interconexões: o vínculo primário: quando a pessoa em desenvolvimento participa ativamente em mais de um ambiente do mesossistema. O vínculo secundário: são as outras pessoas que participam dos mesmos dois ambientes, contudo, ativamente apenas em um deles e no outro participa eventualmente. A comunicação interambiental refere-se às mensagens transmitidas de um ambiente para o outro com a intenção de passar informações para as pessoas do outro ambiente. $\mathrm{O}$ conhecimento interambiental refere-se à informação ou experiência que existe de um ambiente a respeito ao outro.

Um exossistema se refere a um ou mais ambientes que não envolvem a pessoa em desenvolvimento como participante ativo, mas no qual ocorrem eventos que afetam, ou são afetados, por aquilo que acontece no ambiente contendo a pessoa em desenvolvimento. Ele apresenta os mesmos elementos do mesossistema, exceto o vínculo primário. Por sua vez, o macrossistema é o conjunto de micro, meso e exossistema característicos de uma cultura, sub-cultura ou contexto social maior. Com seus recursos, crenças, costumes, oportunidades e estilos de vida próprios. Por fim, o chamado cronossistema envolve uma dimensão temporal, incluindo mudanças significativas centradas no desenvolvimento da pessoa e/ou contexto (Bronfenbrenner, 1996).

A participação de crianças, adolescentes e adultos, como agentes de difusão de diferentes formas de mobilidade ativa, relacionada ao meio ambiente e a saúde no contexto escolar e extraescolar, poderia instigar a participação deles nessas atividades. Poderiam ser desenvolvidas em escolinhas esportivas, em clubes de ciência, nas aulas curriculares, de forma multidisciplinar e interdisciplinar com outras disciplinas curriculares, envolvendo a pedagoga da escola, em programas-projetos de esportes, de ciências, entre ouros, em um contexto maior envolvendo os familiares dos alunos, assim como os órgãos públicos e os privados, ampliando a cultura da mobilidade ativa no estado e no nosso país, por meio de oportunidades e estilos de vida favoráveis ao desenvolvimento de diferentes formas de mobilidade ativa, visando maior efetividade na saúde e no meio ambiente.

Nesse sentido, a ODS 4 (ONU, 2015) trata sobre utilizar instalações físicas para educação, apropriadas e que proporcionem ambientes de aprendizagem seguros e eficazes para todos. Versa também sobre ampliar substancialmente o número de bolsas de estudo para os países em desenvolvimento, bem como propõe aumentar substancialmente o contingente de professores qualificados, inclusive por meio da cooperação internacional para a formação de professores, nos países em desenvolvimento. Desta forma, envolvendo pessoas qualificadas, visando a melhor aplicação educacional, contribuindo com a melhoria das condições da mobilidade ativa, proporcionando melhores condições para a saúde e para o meio ambiente, no ambiente escolar, relacionando com o extracurricular, e demais ambientes que formam o contexto de desenvolvimento humano.

\section{Considerações Finais}

O ambiente de aprendizagem educacional formal e não formal, criando oportunidades, instrução e encorajamento, pode incluir as características do indivíduo, tais como suas capacidades físicas, motoras e intelectuais, para estimulá-lo à 
utilização da mobilidade ativa, atendendo a complexidade, aos equipamentos, aos objetivos e a estruturas de regras da prática da mobilidade ativa, considerando seus benefícios para a saúde e para o meio ambiente.

Incorporar a mobilidade ativa, cada vez mais, no ambiente escolar, em suas diferentes fases, principalmente em se tratando da prática com o uso da bicicleta, torna-se importante. Além disso, é necessário interagir o ambiente educacional formal com o não formal, bem como o ambiente familiar e o poder público, por meio de projetos de mobilidade ativa. Desta forma, temse que estimular a prática da atividade física por meio da mobilidade ativa de forma adequada favorecerá a saúde humana e o meio ambiente.

Compreender quais as características do indivíduo, do ambiente e da tarefa, para intervenções específicas, na mobilidade ativa, tornam-se importante para as decisões a serem tomadas, visando possibilitar decisões bem informadas sobre quais intervenções justificam sua implementação em uma população maior, em se tratando da mobilidade ativa. Mostra-se necessário criar ações pontuais, bem como intervenções que possam ser incorporadas à prática e aos contextos sistêmicos em andamento, nos ambientes do dia a dia das pessoas, são necessárias, favorecendo a sua manutenção por longo tempo. A ampliação dos contextos de ação é relevante, atingindo diferentes ambientes que envolvem a mobilidade ativa e fazem parte da vida do cidadão.

Considerando que se trata de uma necessidade, sugere-se somar o ensino formal com o não formal, com interação da família, fortalecendo o aprendizado. Tratar do assunto envolvendo um ambiente mais amplo, abarcando a relação entre diferentes contextos, é algo fundamental. Por isso, tornam-se importantes projetos envolvendo a relação governo do estado, prefeitura municipal, universidade, educação escolar e comunidade com os Objetivos de Desenvolvimento Sustentável 2030 e a mobilidade ativa, com foco em estratégias inovadoras em educação para a saúde e para o meio ambiente.

Por fim, aponta-se a necessidade de novas pesquisas para maior aproveitamento e aprimoramento da temática mobilidade ativa, abordando outros Objetivos de Desenvolvimento Sustentável 2030 e outras características do indivíduo, do ambiente e da tarefa, e suas interconexões.

\section{Referências}

Bouchard, C., Shepard, R. J., \& Stephens, T. (1993). Physical activity, fitness, and health. Consensus Statement. Champaign: Human Kinetics Publishers.

Bronfenbrenner, U. (1996). A ecologia do desenvolvimento humano: experimentos naturais e planejados: Artes Médicas.

Carvalho, M. L. De., \& Freitas, C. de. (2012). Pedalando em Busca de Alternativas Saudáveis e Sustentáveis. Revista Ciência e Saúde Coletiva, 17 (6), 16171628 .

Conselho Federal de Educação Física. (2012). Resolução CONFEF 229/2012. Dispõe sobre Especialidade Profissional em Educação Física na área de Saúde Coletiva. https://www.confef.org.br/confef/resolucoes/301.

Edra, F. P. M. Cicloturismo: reflexões e experiências contemporâneas: FTH/UFF, $2019 . \quad$ file://C:/Users/55419/Downloads/relatorio voluntario_brasil2017port.pdf. Acesso em: 25 nov. 2020.

Gallahue, D. L., \& Donnely, F. C. (2008). Educação física desenvolvimentista para todas as crianças. (4 ${ }^{\mathrm{a}}$ ed.): Phorte.

Gil, A. C. (2002). Como elaborar projetos de pesquisa. (4ª ed.): Atlas.

Governo do Estado de São Paulo. (2020) Portal de Educação Ambiental. https://www.infraestruturameioambiente.sp.gov.br/cea/portal-de-educacaoambiental/

Governo do Estado do Paraná. (2021). Programa Estadual de Educação Ambiental. http://www.sedest.pr.gov.br/Pagina/Programa-Estadual-de-EducacaoAmbiental.

Heaney, A. K., Carrión, D., Burkart, K., Lesk, C., \& Jack, D. (2019). Climate Change and Physical Activity: Estimated Impacts of Ambient Temperatures on Bikeshare Usage in New York City. Environmental Health Perspectives. 127(3).

Lei 9.795, de 27 de abril de 1999. Dispõe sobre a educação ambiental, institui a Política Nacional de Educação Ambiental e dá outras providências. http://www.planalto.gov.br/ccivil_03/leis/19795.htm

Ministério do Meio Ambiente, Ministério da Educação. Educação ambiental por um Brasil sustentável: ProNEA. Marcos legais e normativos. [recurso eletrônico]. Brasilia, DF. MMA. 
Research, Society and Development, v. 10, n. 6, e47510616052, 2021

(CC BY 4.0) | ISSN 2525-3409 | DOI: http://dx.doi.org/10.33448/rsd-v10i6.16052

Newell, K. M. Constraints on the development of coordination. (1986). In: Wade, M. G., \& Whiting, H. T. A. Motor development in children: aspects of coordination and control. Amsterdam: Nijhoff Publishers.

Newell, K. M., \& Jordan, K. (2007). Task constraints and movement organization: a common language. In: Davis, W. E., \& Broadhead G. D. Ecological task analysis and movement. Champaign: Human Kinetics.

Organização das Nações Unidas. (2015). Nações Unidas - Brasil. Transformando Nosso Mundo: A Agenda 2030 para o Desenvolvimento Sustentável. www.undp.org > dam > brazil > docs > agenda2030

Presidência da República. Lei 9.503, de 23 de setembro de 1997. Institui o Código de Trânsito Brasileiro. http://www.planalto. gov.br/ccivil_03/leis/L9503Compilado.htm.

Relatório Nacional Voluntário sobre os $\quad$ Objetivos de $\quad$ Desenvolvimento

http://www4.planalto.gov.br/ods/publicacoes/relatoriovoluntario_brasil2017port.pdf

Secretaria de Educação Fundamental. Parâmetros curriculares nacionais: Educação física / Secretaria de Educação Fundamental. Brasília: MEC/SEF. http://portal.mec.gov.br/seb/arquivos/pdf/livro 07.pdf.

Waters, E. De., Silva-Sanigorski, A., Burford, B. J., Brown, T., Campbell, K. J., Gao, Y., Armstrong, R., Prosser, L., \& Summerbell, C. D. (2011). Interventions for preventing obesity in children. Cochrane Database of Systematic Reviews, 12 (1871), 1-208.

World Health Organization. (2013). Obesity. Genebra: WHO. < http://www.who.int/topics/obesity/en/ >.

World Health Organization. (2018) Physical activity. Genebra: WHO. https://www.who.int/en/news-room/fact-sheets/detail/physical-activity. 